\title{
Influence of saline based hydroxylethyl starch on umbilical cord blood electrolytes
}

Atsushi Nishigaki Tomoaki Yatabe Masataka Yokoyama

Department of Anesthesiology and Intensive Care Medicine, Kochi Medical School

\section{BackGround}

Hydroxyethyl starch (HES) is widely used to prevent and treatment for spinal induced hypotension in cesarean section. On the other hand, use of saline-based HES might lead to be hyperchloremia. However, influence of salinebased HES on umbilical cord blood electrolyte remain unclear. Therefore, we conducted retrospective analysis.

\section{Method}

Study Design: Retrospective study

Subjective: Consecutive single-pregnancy patients who underwent cesarean section with combined spinal-epidural anesthesia, at no sooner than 37 weeks of gestation (2014/1-2016/3)

Dividing: group A; Received saline-based 6\% HES 130/0.4 group B; HES not administered

Data collection: Age, BMI, Gestational week, Time to delivery from operating room admission, Infusion volume, Blood loss, Urine volume, Apgar score at 1 and 5 min, Umbilical cord blood $\left(\mathrm{Cl}^{-}, \mathrm{Na}^{+}, \mathrm{K}^{+}, \mathrm{pH}\right.$,base excess $)$ Definition: The difference of $2.5 \mathrm{mmol} / \mathrm{l}$ or more as clinically significant considering $15 \%$ of the normal range Analysis: The Mann-Whitney U test. P values less than 0.05 were considered statistically significant.

\begin{tabular}{|c|c|c|c|c|}
\hline & & $\begin{array}{c}\text { Group A } \\
(\mathbf{N}=\mathbf{4 3})\end{array}$ & $\begin{array}{c}\text { Group B } \\
(\mathbf{N}=\mathbf{5 0})\end{array}$ & P valu \\
\hline Age & (years & $34 \pm 5$ & $32 \pm 6$ & 0.07 \\
\hline Height & $(\mathbf{c m}$ & $157 \pm 5$ & $156 \pm 5$ & 0.64 \\
\hline Weight & (kg & $59 \pm 9$ & $61 \pm 10$ & 0.50 \\
\hline Body mass index & $(\mathrm{kg} / \mathrm{m}$ & $24 \pm 4$ & $25 \pm 4$ & 0.44 \\
\hline Gestational week & (week & $38 \pm 1$ & $38 \pm 1$ & 0.08 \\
\hline Duration of operation & $(\min )$ & $75 \pm 19$ & $78 \pm 19$ & 0.45 \\
\hline Duration of anesthesia & $(\min )$ & $99 \pm 22$ & $102 \pm 21$ & 0.51 \\
\hline Time to delivery & $(\mathrm{ml})$ & $57 \pm 14$ & $56 \pm 14$ & 0.74 \\
\hline Total infusion & $(\mathrm{ml})$ & $2247 \pm 523$ & $2101 \pm 578$ & 0.21 \\
\hline Blood loss & $(\mathrm{ml})$ & $966 \pm 443$ & $987 \pm 460$ & 0.82 \\
\hline Urine volume & (ml) & $335 \pm 209$ & $383 \pm 299$ & 0.38 \\
\hline
\end{tabular}

\section{Results}

$\checkmark$ We analyzed data of 93 patients;

(Group A: 43 patients, B: 50 patients)

Background were not significantly different between two groups (Table 1).

Infusion volume until delivery were similar. $461 \pm 167 \mathrm{ml}$ of saline-based HES was administered in group A (Table 2).

The umbilical cord chloride level at delivery was significantly higher in group A.(Table 2). No differences were observed in the Apgar score and other umbilical cord laboratory data at delivery $\left(\mathrm{Na}^{+}, \mathrm{K}^{+}, \mathrm{pH}, \mathrm{BE}\right)$ (Table.2).

\section{Discussion}

Previous studies showed use of saline-based HES influenced $\mathrm{pH}$ and $\mathrm{Cl}^{-}$(2-3 mmol/l in $\mathrm{Cl}^{-}$level and 0.03 in $\mathrm{pH}$ ) in major surgery ${ }^{1,2}$. Our study indicated that absolute difference value in chloride level was only $1 \mathrm{mmol} / \mathrm{l}$. In terms of the risk-benefit balance, anesthesiologists may consider $500 \mathrm{ml}$ of saline-based HES as the upper limit for routine use .

\begin{tabular}{|c|c|c|c|c|}
\hline & & $\begin{array}{l}\text { Group A } \\
(\mathbf{N}=\mathbf{4 3})\end{array}$ & $\begin{array}{c}\text { Group B } \\
(\mathbf{N}=\mathbf{5 0})\end{array}$ & P value \\
\hline Infusion until delivery & $(\mathbf{m l})$ & $1316 \pm 384$ & $1176 \pm 444$ & 0.11 \\
\hline Saline-based HES 130/0.4 & (ml) & $461 \pm 167$ & $\mathbf{0}$ & - \\
\hline Bicarbonate Ringer's solution & $(\mathbf{m l})$ & $470 \pm 410$ & $541 \pm 410$ & 0.41 \\
\hline Acetate Ringer's solution & $(\mathrm{ml})$ & $294 \pm 332$ & $541 \pm 389$ & 0.002 \\
\hline Saline & $(\mathbf{m l})$ & $91 \pm 29$ & $94 \pm 24$ & 0.55 \\
\hline Apgar score $1 \mathrm{~min}$ & & $7.9 \pm 0.7$ & $7.9 \pm 1.0$ & 0.94 \\
\hline Apgar score 5 min & & $8.8 \pm 0.5$ & $8.6 \pm 0.9$ & 0.21 \\
\hline \multicolumn{5}{|l|}{ Umbilical cord data on delivery } \\
\hline pH & & $7.32 \pm 0.04$ & $7.30 \pm 0.05$ & 0.12 \\
\hline Base excess (mmol/l) & & $-2.8 \pm 1.6$ & $-2.8 \pm 1.6$ & 0.94 \\
\hline $\mathrm{Na}^{+}(\mathbf{m m o l} / \mathbf{l})$ & & $136 \pm 2$ & $136 \pm 1$ & 0.69 \\
\hline $\mathbf{K}^{+}(\mathbf{m m o l} / \mathbf{l})$ & & $3.9 \pm 0.4$ & $4.0 \pm 0.3$ & 0.69 \\
\hline $\mathrm{Cl}^{-}(\mathbf{m m o l} / \mathbf{l})$ & & $108 \pm 2$ & $107 \pm 2$ & 0.02 \\
\hline
\end{tabular}

(Table. 2) Infusion and laboratory data

\section{Conclusion}

